\title{
A THEOREM ON THE EXISTENCE AND UNIQUENESS OF SOLUTIONS FOR ABSTRACT, QUASI-LINEAR DIFFERENTIALL EQUATIONS
}

1. Derivatives, integrals, limit conditions Assume that we are given:

a) real linear spaces: $L^{1}$ and $\left.L^{0}, L^{1} \subset L^{0}, *\right)$

b) m derivatives: i.e. Iinear operations

$$
S_{1}, S_{2}, \ldots, S_{m}: L^{1} \longrightarrow L^{0}
$$

from the space $I^{1}$ onto the space $I^{0}$, so that

$$
I^{1}=\left\{x \in L^{0}, \wedge_{\dot{i}} S_{i} x \in L^{0}\right\} .
$$

The space $\mathrm{L}^{2}$ is defined as a subspace $L^{1}$ such that

$$
\begin{aligned}
& S_{j_{j}}: I^{2} \longrightarrow I^{1} \quad \text { (onto) } \\
& I^{2}=\left\{x \in L^{0}, \bigwedge_{i, j} S_{i} S_{j} x \in L^{0}\right\} .
\end{aligned}
$$

*) The symbols $\mathrm{L}^{0}, \mathrm{I}^{1}$ denote any real linear spaces such that $L^{1} \subset L^{0}$ (the other notion of functional spaces $L^{p}=$ $=\left\{x:\left(\left.\int_{0}^{1}|x(t)|\right|^{p} d t\right)^{\frac{1}{p}<+\infty}\right\}$ is not used $)$. 
By Induction we define the space $I^{\text {n }}$ as

$$
L^{n}=\left\{x \in L^{0}: \underset{\left(i_{1}, \ldots, I_{n}\right)}{\bigwedge} s_{i_{1}} s_{1_{2}} \ldots s_{I_{n}} x \in I^{0}\right\} .
$$

So we have

$$
\ldots \subset \mathrm{L}^{\mathrm{n}} \subset \mathrm{L}^{\mathrm{n}-1} \subset \ldots \subset \mathrm{L}^{1} \subset \mathrm{L}^{0} .
$$

Let us assume that for derivatives $S_{i}$ there exist integrals 1.e. Inear operations

$$
\hat{i} T_{i}\left(q_{i}\right): L^{0} \longrightarrow L^{1}
$$

(the $g_{1}$ indices belongs to sets $Q_{1}$ ), such that we have

$$
\hat{i} S_{i} T_{i}\left(q_{i}\right)=i d{ }_{I} 0
$$

and the corresponding limit conditions hold

$$
s_{i}\left(q_{1}\right) x=x-T_{1}\left(q_{i}\right) s_{i} x
$$

We have for $c \in$ ker $S$

$$
s_{i}^{2}\left(g_{i}\right) c=s_{i}\left(g_{i}\right) c=c . \quad(\text { see }[2]) .
$$

We assume that various derivatives and integrals do commute

$$
\begin{gathered}
S_{i} S_{j} x=S_{j} S_{i} x, \quad \text { for } x \in L^{2}, \quad i, j=1,2, \ldots, m . \\
T_{i}\left(g_{i}\right) T_{j}\left(q_{i}\right) f=T_{j}\left(g_{j}\right) T_{i}\left(g_{i}\right) f, \text { for } f \in L^{0}, \quad i \neq j . \\
S_{i} T_{j}\left(g_{j}\right) x=T_{j}\left(g_{j}\right) S_{i} x, \text { for } x \in I^{1}, \quad i \neq j .
\end{gathered}
$$


It is then easy to prove, using the definition of limit condition, that we have

$$
\begin{aligned}
& s_{i} a_{j}\left(q_{j}\right) x=\theta_{j}\left(q_{j}\right) s_{i} x, \quad \text { for } x \in I^{2}, i \neq j, \\
& T_{i}\left(q_{i}\right) g_{j}\left(q_{j}\right) x=\sigma_{j}\left(q_{j}\right) T_{i}\left(q_{i}\right) x, \text { for } x \in I^{1}, \quad i \neq j, \\
& s_{i}\left(g_{i}\right) s_{j}\left(g_{j}\right) x=s_{j}\left(g_{j}\right) s_{i}\left(q_{i}\right) x, \text { for } x \in I^{2}, \quad i \neq j \text {. }
\end{aligned}
$$

(see [3]).

2. Differential equations. Singular integrals

We assume that $L^{0}$ is a Banach space and that integrala $T_{i}\left(q_{i}\right)$ are continuous in $L^{0}$.

Let us consider the equation

$$
\mathcal{F}\left(x, s_{1} x, \ldots, s_{m} x, s_{i_{1}} \cdots i_{\alpha} x, \bar{f}\right)=0,
$$

where $\alpha<\mathrm{n}, \mathcal{F}$ is a function of variables

$$
x, s_{1} x, \ldots, s_{m} x, s_{i_{1}} \cdots i_{\alpha} x, \vec{f} .
$$

The sjmbol $\vec{f}_{D_{f}}=\left\{S_{i_{1}}, \ldots i_{n} x\right\}$ denotes a vector $\vec{f}$ with coordinates $f_{i_{1}}, \cdots i_{n}=s_{i_{1}}, \ldots i_{n} x$.

Let us introduce the notations:

$$
\begin{aligned}
& s_{I}=s_{i_{1}}, \ldots{i_{k}}_{k}=s_{i_{1}} \cdot s_{i_{2}} \ldots s_{i_{k}}, \quad I=\left(i_{1}, \ldots, i_{k}\right) \\
& s_{I}=s_{i_{1}}, \ldots i_{\alpha}=s_{i_{1}} \cdot s_{i_{2}} \ldots s_{i_{\alpha}}, \quad I=\left(i_{1}, \ldots, i_{\alpha}\right) \\
& T_{I}\left(q_{I}\right)=T_{i_{1}}, \ldots{i_{i}}_{k}(q)=T_{i_{1}}\left(g_{i_{1}}\right) \ldots T_{i_{k}}\left(q_{i_{k}}\right) \text {, } \\
& T_{L}\left(q_{I}\right)=T_{i_{1}}, \ldots{i_{\alpha}}(q)=T_{i_{1}}\left(q_{i_{1}}\right) \ldots T_{i_{\alpha}}\left(q_{i_{\alpha}}\right) \text {, } \\
& \vec{f}=\vec{S}^{n} x=\left\{s_{i_{1}}, \cdots{\dot{i_{n}}}_{n} x\right\}=\left\{f_{i_{1}}, \cdots{i_{n}}\right\} \text {, for } x \in L^{n+1} \text {, } \\
& s_{L} x \in s_{L}\left(L^{n+1}\right), \quad \vec{S}^{n} x \in \bigcap_{J} s_{J}\left(L^{n+1}\right) \text {, }
\end{aligned}
$$

where $J=\left(i_{1}, i_{2}, \ldots, i_{n}\right)$. 
D I Inition 1. If an element

$$
W:\left\{s_{J} W\right\}=0, \quad 1 \leqslant i_{1} \leqslant \ldots \leqslant i_{n} \leqslant m
$$

is the solution of the equation

$$
F\left(W, s_{1} w, \ldots, s_{m} w, s_{L} w, \ldots,\{0\}\right)=0
$$

it will be called a singular integral of the equation (2.1).

De $f$ in $i t i \circ n$ 2. If an element $x$ is a nongin-. gular integral: $1 . e$.

when $\left\{s_{J} x\right\} \neq 0$, then for

$$
\mathcal{F}\left(x, s_{1} x, \ldots, s_{I} x, \ldots,\left\{s_{J} x\right\}\right)=0
$$

$$
\begin{array}{ll}
a \underset{D f}{=}\left\{x, \ldots, S_{L} x, \ldots\right\}, & G_{a}=\{a\} \subset \underset{L}{X} S_{L}\left(I^{n+1}\right) \\
b \underset{D f}{b}\left\{S_{J} x\right\}, & G_{b}=\{b\} \subset 2,
\end{array}
$$

where $z=\underset{J}{X} L^{1}$, we have

$$
F(a, b)=0, \quad\left(G_{a} \times G_{b}\right) \subset\left(x_{L} S_{L}\left(I^{n+1-k}\right) \times z\right),
$$

with norms

$$
\|b\|_{*}=\sup _{J}\left\|S_{j} x\right\|,\|a\|_{*}=\sup _{D f}\|x\|, \ldots,\left\|S_{L} x\right\|, \ldots,
$$

in products of some Banach spaces.

Now we will give a theorem about the equivalence of the implicite equation (2.2) and an explicite equation for the $n$-th gradient $S^{n} x$ in certain neighbourhood of nonsingular integral.

We will use the theorem about implicite functiolns. 
Let us consider the equation

$(2.3)$

$$
F(a, b)=0
$$

with an additional condition
$(2.4)$
$g(a, b)=0$

Let

$$
\phi(a, b) \underset{\text { Df }}{=}(\mathcal{F}(a, b), g(a, b)) .
$$

We assume the function $\phi(a, b)$ has in a neighbourhood continuous Frechet.'s derivative $\mathcal{F}_{b}^{i}\left(a_{0}, b_{0}\right)$, and that there exists $\left(F_{b}^{\prime}\left(a_{0}, b_{0}\right)\right)^{-1}$. When there are neighbourhoods $U_{a_{0}} \subset G_{a}$ and $\mathrm{W}_{\mathrm{b}} \subset \mathrm{G}_{\mathrm{O}}$, and a continuous operation

$$
\hat{I} \underset{D f}{=}\left\{f_{J}\right\}: U_{a_{0}} \longrightarrow w_{b_{0}},
$$

such that the equation

$$
b=f(a)
$$

is equivalent to the equations

$$
\begin{array}{ll}
F(a, b)=0, & b_{0}=I\left(a_{0}\right) \\
g(a, b)=0, & g\left(a_{0}, b_{0}\right)=0 .
\end{array}
$$

By the properties of elements $a$ and $b$ we obtain an explicite differential equation of the $n$-th order

$$
\left\{s_{J} x\right\}=f_{J}\left(\ldots, s_{I} x, \ldots\right) \quad \alpha<n
$$

equivalent to the implicite differential equation (2.1) in the neighbourhood $\mathrm{u}_{\mathrm{a}_{0}}$. 
$D \in f i n i t i o n$ 3. The points $(a, b)$ such that $(2.6)$

$$
F(a, b)=0, \quad g(a, b)=0
$$

and that $\left(f_{b}^{\prime}(a, b)\right)^{-1}$ does not exist or is not continuous, will be called degenerate points of the equation (2.1).

In the case of nondegenerate points $\left(a_{0}, b_{0}\right)$ there is an equivalent explicite equation $(2.6)$ with the continuous right side for $a \in U_{a_{0}}$ in a neighbourhood of $\left(a_{0}, b_{0}\right)$.

We shall further consider an explicite differential equation.

3. Necessary condition for the existence of solutions for a differential equation

Let us consider the equation

$$
\vec{S}^{n} x=\vec{f}(a),
$$

where $\vec{f}$ is a continuous function,

$$
a \underset{D f}{=}\left\{x, \ldots, S_{L} x, \ldots\right\}, \alpha<n
$$

$$
s(g) a \underset{D f}{=}\left\{g(q) x, \ldots, g(g) s_{I} x, \ldots\right\} \underset{D f}{=}\left\{a_{0}(x)\right\} .
$$

By relation (3.2) we can develope $x$ in Taylor's formula $\left.(\text { see }[3])^{*}\right)$

$$
x=w_{n-1}(q) x+d^{n}(q) s^{n} x
$$

*) The Taylor formula for m derlvatives $s_{1}, s_{2}, \ldots, s_{m}$ can be obtained also by formulas from [1] and [4], [5]. 
A theorem on the existence

7

where

$$
W_{n-1}(q) x=\sum_{L} T_{L}(g) s(g) s_{I} x, \quad L=\left\{i_{1}, i_{2}, \ldots, i_{\alpha}\right\}, \quad \alpha<n .
$$

Let

(3.4) $\left\|d^{n}(q)\right\| \underset{D f}{=} \delta_{H}$,

where $H=\left\{x, q, W_{n-1}(q) x, a\right\}$.

I em ma. Let

$$
\left\|x-W_{n-1}(q) x\right\| \leqslant \alpha,
$$

where

$$
\alpha \underset{D f}{=} \delta_{H}\left\|S^{n} x\right\|
$$

Pr o $\circ . \quad B y(3.3)$ we have

$$
\left\|x-W_{n-1}(q) x\right\|=\left\|d^{n}(q) S^{n} x\right\| \leqslant \delta_{H i}\left\|S^{n} x\right\| \leqslant \alpha .
$$

I em ma. If an element of the equation (3.1) with limit condition (3.2) in a set $G$ is a nonsingular solution of the equation (3.5), then

$$
\begin{aligned}
& \sup _{x \in K\left(W_{n-1}(q), x, \varepsilon\right)} \frac{\left\|x-W_{n-1}(q) x\right\|}{\left\|\inf _{Y} \vec{f}(a(g))\right\|}=\delta_{H} \\
& Y=\left\{y:\left\|y-W_{n-1}(q) x\right\|=\left\|x-W_{n-1}(q) x\right\|,\left\|y-W_{n-1}(q) x\right\|<\varepsilon\right\} . \\
& \text { P r o o } f \cdot 1^{0} \text { Because } f \text { is a continuous function, } \\
& \text { we have } \\
& \left.\left\|\vec{s}^{n} x\right\|=\| \vec{f}(x)\right) \| \neq 0
\end{aligned}
$$


8

r.Bittnar, 4. Smentêk

in the neighbourhood

$$
\begin{array}{r}
\|x-\|_{n-1}(q) x \| \leqslant \alpha . \\
\left\|x-w_{n-1}(q) x\right\| \leqslant \delta_{H}\left\|\vec{s}^{n} x\right\|, \text { so that } \\
\frac{\left\|x-n_{n-1}(q) x\right\|}{\| \vec{s}^{n} x} \leqslant \delta_{H} .
\end{array}
$$

For $0<\varepsilon<\delta_{H}$ there exists an $x_{\varepsilon}$ such that

$$
\delta_{H}-\varepsilon \leqslant \frac{\left\|x_{\varepsilon}-W_{n-1}(q) x_{\varepsilon}\right\|}{\left\|\vec{s} x^{n}\right\|} \leqslant \delta_{i H},
$$

where $x \in K^{\prime} \underset{D f}{=} K\left(W_{n-1}(q) x, \varepsilon\right) \cdot$ But

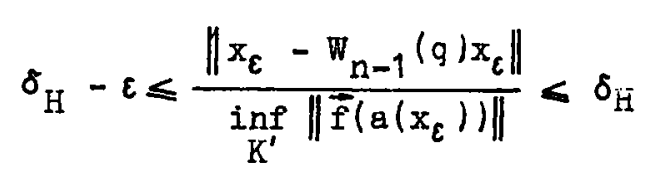

and

$$
\delta_{H}-\varepsilon \leqslant \sup _{k^{\prime \prime}} \frac{\left\|x-W_{n-1}(g) x\right\|}{\inf _{Y}\|\vec{f}(g(y))\|} \leqslant \delta_{H^{\prime}} \cdot
$$

If $(3.6)$ is true then

$$
\frac{\left\|x-W_{n-1}(g) x\right\|}{\inf _{Y}\|\vec{f}(a(g))\|}<\delta_{H}
$$

$-794-$ 
A theorem on the existence

9

and

$$
\frac{\left\|x-w_{n-1}(q) x\right\|}{\| \vec{f}(a(x) \|} \leqslant \delta_{H}
$$

so that $\left\|x-w_{n-1}(q) x\right\| \leqslant \delta_{H}\left\|S^{n} x\right\|$.

4. A theorem about the existence of a solution for a disferential equation

The $\mathrm{T}$ em 1. (i) Consider two assumptions:

(A) there exists a solution $x$ of the equation

$(4.1)$

$$
\vec{S}^{n} x=\vec{f}(a(x))
$$

with limit conditions

and

$$
W_{n-1}(q) x=\xi_{q}^{n-1}
$$

$(4 \cdot 3)$

$$
\left\|x-W_{n-1}(g) x\right\|_{L^{0}} \leqslant \delta\|\vec{f}(a(x))\|_{L^{0}}
$$

(B) there exist solutions $x_{i}$ of the equations

$(4.4)$

$$
\overrightarrow{S_{i}^{n} x_{i}}=\overrightarrow{f^{i}}\left(a\left(x_{i}\right)\right), \quad 0 \leqslant i<N
$$

with limit conditions

$$
w_{n-1}\left(q_{1}\right) x_{i}=\xi_{q_{i}}^{n-1}, \quad 0<1 \leqslant \mathbb{N}
$$

and

$(4.6)$

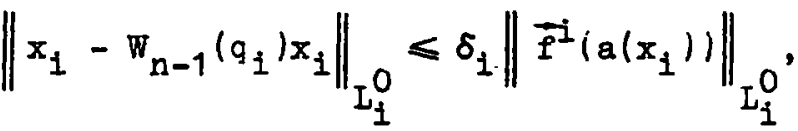

$$
\begin{aligned}
& \text { - } 795-
\end{aligned}
$$


where

$\left\{\left(x, q, \mathbb{W}_{n-1}(q) x, a(x)\right\} \in H=\left\{\left(x_{1}, q_{1}, \mathbb{W}_{n-1}\left(q_{1}\right) x:, a\left(x_{1}\right)\right\} \in H_{1}\right.\right.$.

Moreover, let $0<\delta_{1}=\delta_{\mathrm{H}}<+\infty, \quad 0 \leqslant 1 \leqslant N$ and $\sup _{H_{1}}\left\|\vec{f}^{-1}\left(a\left(x_{1}\right)\right)\right\|_{L_{1}^{0}}=\mu_{1}$.

We assume that (A) is equivalent with $(B)$.

(ii) Let us denote

$$
\left.A_{i} x_{i}=W_{n-1}\left(q_{i}\right) x_{1}+d^{n}\left(q_{1}\right) \vec{f}^{i}\left(a i x_{i}\right)\right)
$$

where $d^{n}\left(q_{1}\right): I_{1}^{0} \longrightarrow I_{1}^{0}$

$$
\left\{x_{1}, q_{1}, w_{n-1}\left(q_{1}\right) x_{1}, \quad a\left(x_{1}\right)\right\} \in H_{1} .
$$

We suppose that the set

$$
\begin{gathered}
\dot{\bar{w}}_{i}=A_{i}\left(v_{i}\right), \text { where } x_{i} \in \mathbb{K}\left(w_{n-1}\left(q_{i}\right) x_{i}, \quad \delta_{i}\left(\mu_{i}\right)\right), \\
v_{1}=w_{n-1}\left(q_{i}\right) x_{i}=\xi_{q_{i}}^{n-1}
\end{gathered}
$$

is compact for $i=1,2, \ldots, N$.

It follows from (1) and (ii) that there exists a solution of the equation (4.1).

P $r \circ \circ f$. The set

$$
v_{i}=\left\{k\left(w_{n-1}\left(g_{1}\right) x_{i}, \delta_{i}\left(\mu_{i}\right)\right)\right\} \cap\left\{w_{n-1}\left(q_{1}\right) x_{i}=\xi_{q_{i}}^{n-1}\right\}
$$

is a convex set.

$$
\text { For }\left\|x_{i}-W_{n-1}\left(g_{i}\right) x_{i}\right\| \leqslant \delta_{G_{i}} \mu_{i}
$$

we have 


$$
\begin{aligned}
& \left\|A_{i} x_{1}-w_{n-1}(q) x_{i}\right\|=\left\|d^{n}\left(q_{1}\right) \vec{f}^{-1}\left(a\left(x_{1}\right)\right)\right\| \leqslant \\
& \leqslant\left\|d_{1}^{n}\left(g_{1}\right)\right\| \cdot\left\|\overrightarrow{f^{i}}\left(a\left(x_{i}\right)\right)\right\| \leqslant \delta_{G_{i}} \mu_{1}=\delta_{i} \mu_{1},
\end{aligned}
$$

or $A_{i}\left(V_{i}\right) \subset \bar{W}_{i}$ and set $\bar{W}_{i}$ is compact.

The assumptions of Schauder's theorem are fulfilled and there exists $x_{i}$ such that

$$
A_{i} x_{1}=x_{1}
$$

i.e. there exist solutions of the equation (4.7).

We have

$$
\begin{gathered}
\vec{s}_{i}^{n} x_{i}=\vec{f}^{i}\left(a\left(x_{i}\right)\right) \\
w_{n-1}\left(q_{i}\right) x_{i}=\xi_{q_{i}}^{n-1} \\
\text { for } \| x_{i}-w_{n-1}\left(q_{i} \mid x_{i}\left\|_{L} \leqslant \delta_{i}\right\| \vec{f}^{i}\left(a\left(x_{1}\right)\right) \|_{L_{i}^{0}}\right.
\end{gathered}
$$

5. The uniqueness of a solution of the equation (4.1)

The $\mathrm{r} \theta \mathrm{m}$ 2. Let $A_{i}$ be defined in (4.7). Suppose that

$$
x_{p+1}^{i}=A_{i}\left(x_{p}^{i}\right), \quad x_{0}^{i}=w_{n-1}(q) x_{i}
$$

Elements $x_{p}^{i}$ for the operations $A_{i}$ are obtained by the succesive approximations method. We assume the conditions:

$$
\sum_{n=1}(\alpha \theta)^{[n]}<+\infty,
$$

where $(\alpha \theta)^{[n]}$ denotes the $n-t h$ iteration of the function $(a \theta)$, 


$$
\begin{gathered}
(\alpha \theta)^{[n+1]} \underset{D f}{=}(\alpha \theta)(\alpha \theta)^{[n]} \\
\left\|f\left(a\left(\bar{x}_{i}\right)\right)-f\left(a\left(x_{i}^{*}\right)\right)\right\| \leqslant \theta\left\|\bar{x}_{i}-x_{i}^{*}\right\|, \\
\frac{1}{N} \sum_{I}\left\|T_{i}(q)\right\|\left\|A_{I}^{-1}(x) s(q)\right\|_{D f}=\alpha<1, \\
R \underset{D f}{=}\left\|x_{1}-x_{0}\right\| .
\end{gathered}
$$

When there does exist for $R \in(0,+\infty)$ the unique solution of the equation (4.1).

and

Prool. We have, for $\bar{x}_{i}=\lim _{p \rightarrow \infty} x_{P+1}^{i}, \bar{x}_{i}=A_{i}\left(\bar{x}_{i}\right)$

$$
\begin{gathered}
\left\|\bar{x}_{i}-x_{i}^{*}\right\| \leqslant \alpha,\left\|f\left(a\left(\bar{x}_{i}\right)\right)-f\left(a\left(x_{i}^{*}\right)\right)\right\| \leqslant(\alpha \theta)\left\|\bar{x}_{i}-x_{i}^{*}\right\| \\
\leqslant \ldots \leqslant(\alpha \theta)^{[n]}(k) \longrightarrow 0 .
\end{gathered}
$$

But $\sum_{n=1}^{\infty}(\alpha \theta)^{[n]}(k)<+\infty$, so that

$$
\left\|\bar{x}_{i}-x_{i}^{*}\right\| \longrightarrow 0 \text {, or }\left\|\bar{x}_{i}=x_{i}^{*}\right\| \text {. }
$$

6. A quasi-linear equation of the $\{i, n)$ order

Let us consider the derivative

(6.1)

$$
\sum_{(1, n)} u=\left.\right|_{z=x} \sum_{I} s_{I}(z) s(z) s_{I}^{x} u
$$

where $I=\left(i_{1}, \ldots, i_{k}\right), \quad I \leqslant k \leqslant n, \quad x \in \bar{\Omega}$, operations $\dot{h}_{I}(z)$ are continuous end have continuous inverses in a donain $\bar{\Omega} \subset \theta^{m}$. 
There is $A_{I}(z): \operatorname{Ker} S \longrightarrow \operatorname{Ker} S$ for $z \in \bar{\Omega}$. We understand the symbol $\left.\right|_{z=x} \sum_{I} A_{I}(z) s(z) s_{I}^{x} u$ in such a way that first of all we find the derivative $s_{I}^{x} u$ for the function $u=\{u(x)\}$ then we take limit condition $s(z) S_{I}^{x} u$, multiply $A(z) s(z) S_{I}^{x} u$ and repeat substitution $\left.\right|_{z=x} \sum_{I} A_{I}(z) s(z) S_{I}^{x} u$. $R \otimes m a r k$. For example substitute for $u=\{u(x)\}$, $x \in \bar{\Omega}, \quad u=u(k), \quad k \in \mathbb{N}^{m}$

$$
\begin{aligned}
& A_{I}(x) \frac{\partial u}{\partial x}=\left.\right|_{z=x} \frac{\partial u}{\partial x} \\
& a_{I}(k) \Delta_{k} u=\left|\begin{array}{ll}
z=k & a_{I}(z)
\end{array}\right|_{k=z} \Delta_{k} u \cdot
\end{aligned}
$$

Definition. The equation

$$
\sum_{(1, n)} u=\mu u+f\left(x, u, S_{I}^{x} u\right), \quad x \in \bar{\Omega}
$$

$I=\left(i_{1}, i_{2}, \ldots i_{\alpha}\right)$ will be called a quasi-linear equation of the order $(1, n)$.

Let us consider for the equation (6.2) a limit condition

$$
\begin{aligned}
\lambda(I, n) u & =\left.\right|_{z=p} \sum_{L} B_{L}(z) s(z) S_{L}^{x} u+\left.\right|_{z=p} h(z) u= \\
& =\varphi\left(p, u, S_{J}^{x} u\right),
\end{aligned}
$$

where $\alpha<I, \quad p \in \bar{k}, J=\left(j_{1}, \ldots, j_{\beta}\right), \beta<\alpha, B_{L}$ are continuaus and have continuous inverses in a set $\overline{\mathrm{k}} \subset \bar{\Omega} \subset \theta^{\mathrm{m}}$. 
Theorem 3. If

$$
\sum_{I} F_{I}(p)=1, \quad \sum_{I} G_{I}(x)=1, \quad F_{I} \geqslant 0, G_{I} \geqslant 0
$$

then the element

$$
\begin{aligned}
(6.4) u & =\left.\sum_{I}\left(\left.T_{L}^{x}(z)\right|_{z=p} F_{L}(z) B_{I}^{-1}(z)\right)\right|_{p=z}\left(\varphi\left(p, u, S_{L}^{x} u\right)-h(p) u\right)+ \\
& +\left.\sum_{I}\left(\left.T_{I}^{x}(z)\right|_{z=x} G_{I}(z) A_{I}^{-1}(z)\right)\right|_{x=z}\left(\mu u+f\left(x, u, S_{L}^{x} u\right)\right) \underset{D f}{=\phi(u)}
\end{aligned}
$$

with limit condition (6.3) is a solution of the equation (6.2).

$P$ I $0.0 \mathrm{f}$. We shall prove that the element $(6.4)$ is a solution of the equation (6.2).

We have

(a)

$$
\begin{aligned}
& \left.\left|z=x \sum_{I} A_{I}(z) s(z) S_{I}^{x} \sum_{I} T_{I}^{x}(z)\right|_{z=p} F_{L}(z) B_{p}^{-1}(z)\right|_{p=z} \cdot \\
& \cdot\left(\varphi\left(p, u, s_{L}^{x} u\right)-h(p) u\right)=0,
\end{aligned}
$$

because $I=\left(i_{1}, \ldots, i_{k}\right), \quad I=\left(i_{1}, \ldots, i_{\alpha}\right), \alpha<I \leqslant k<n$, and in the expression $S_{I}^{X}$ the derivatives are of higher order than the order of integrals in the expression in $T_{L}$.

(b) $\left.\left.\quad \sum_{z=x} \sum_{I} A_{I}(z) B(z) S_{I}^{x} \sum_{I^{\prime}} T_{I^{\prime}}^{X}(z)\right|_{z=x} G_{I^{\prime}}(z) A_{I^{\prime}}^{-1}(z)\right|_{X=z}$

$$
\text { - }\left(\mu u+f\left(x, u, S_{L}^{x} u\right)\right)=\mu u+f\left(x, u, S_{L}^{x} u\right),
$$

because 


$$
\begin{aligned}
& \left.\left.\left.\right|_{z=x} s(z) S_{I}^{x} \sum_{I^{\prime}}{ }_{I_{I^{\prime}}}^{X}(z)\right|_{z=x} G_{I^{\prime}}(z) A_{I^{\prime}}^{-1}(z)\right|_{X=z}= \\
& = \begin{cases}0, \text { for } I \neq I^{\prime} \\
G_{I}(x) A_{I}^{-1}(x), & \text { for } I=I^{\prime}\end{cases}
\end{aligned}
$$

and $\sum_{I} G_{I}(x)=1$.

From (a) and (b) we have the equation (6.2). We will prpve that the limit condition $(6.3)$ is fulfilled. We have

$$
\begin{aligned}
& \left.\left.\right|_{z=p} \sum_{I} B_{I^{\prime}}(z) s(z) S_{I}^{x} \sum_{I^{\prime}}{ }_{I_{I}^{\prime}}^{x}(z) F_{I^{\prime}}(z) B_{I^{\prime}}^{-1}(z)\right|_{p=z} \\
& \cdot\left(\varphi\left(p, u, S_{L^{x}}^{x}\right)-h(p) u\right)+h(p) u=\varphi\left(p, u, s_{I}, u\right),
\end{aligned}
$$

because

$\left.\left.\right|_{z=p} s(z) S_{I}^{X} \sum_{I^{\prime}} T_{I^{\prime}}^{X}(z) F_{I^{\prime}}(z) B_{I^{\prime}}^{-1}(z)\right|_{p=z}=\left\{\begin{array}{l}0 \text { for } I \neq I^{\prime} \\ F_{L}(p) B_{I}^{-1}(p) \text { for } L=I^{\prime}\end{array}\right.$

and $\sum_{I} F_{L}(p)=1$.

(d)

$$
\begin{aligned}
& \left.\left.\sum_{I} B_{L}(z)\right|_{z=p} s(z) S_{I}^{x} \sum_{I} T_{I}^{x}(z) G_{I}(z) A_{I}^{-1}(z)\right|_{x=z} \cdot \\
& \cdot\left(\mu u+f\left(x, u, S_{I}^{x} u\right)\right)=0
\end{aligned}
$$

and the orders of derivatives in $S_{L}^{x}$ are lower then the order of integrals in $\mathrm{T}_{I}^{X}$. Then $s(z) \mathrm{S}_{\mathrm{L}}^{\mathrm{X}_{\mathrm{T}}^{\mathrm{X}}}(\mathrm{z})=0$.

From (c) and (d) we obtain that the limit condition (6.3) for the equation $(6.4)$ is fulfilled. 
$\mathrm{R} e \mathrm{mark}$. It results from $1^{\circ}$ and $2^{\circ}$ that

(a) $\left.\quad \sum_{I} T_{L}^{X}(z) F_{L}(z) B_{L}^{-1}(z)\right|_{z=p}\left(\varphi\left(p, u, S_{L}^{X} u\right)-h(p) u\right)$

is a constant for the derivative $(6.1)$.

( $\beta$ )

$$
\text { The operation }\left.\sum_{I} T_{I}^{X}(z) G_{I}(x) A_{I}^{-1}(z)\right|_{x=z}
$$

is the integral for the derivative (6.1).

$(\gamma)$ By $(\alpha)$ and $(\beta)$ we have

$$
\begin{array}{ll}
\sum_{(1, n)} \phi\{f(x)\}=\{f(x)\}, & \lambda_{(1, n)} \phi\{f(x)\}=0, \\
\sum_{(1, n)}\{\varphi(p)\}=0, & \lambda_{(1, n)}\{\varphi(p)\}=\{\varphi(p)\} .
\end{array}
$$

$T$ b $\theta 0 . \mathrm{r} \theta \mathrm{m}$ 4. If the functions $f$ and $\varphi$ finlfill Lipschitz's condition

$$
\begin{aligned}
& \text { (i) }\left\|f\left(x, u, S_{L}^{x} u\right)-f\left(x, v, S_{L}^{x} v\right)\right\| \leqslant M_{0}^{f}\|u-v\|+\sum_{I} M_{L}^{f}\left\|S_{L}^{x} u-S_{I}^{x} / v\right\|, \\
& M_{0}^{f} \geqslant 0, \quad M_{L}^{f} \geqslant 0, \quad M_{0}^{f}+\sum_{I} M_{L}^{f}>0,
\end{aligned}
$$$$
(6.6)(i i)\left\|\varphi\left(p, u, S_{I}^{x} u\right)-\varphi\left(p, v, S_{L}^{x} v\right)\right\| \leqslant \mathbb{M}_{0}\|u-v\|+\sum_{I} \mathbb{M}_{L}\left\|S_{L}^{x} u-S_{L}^{x} v\right\|,
$$$$
\mathbb{M}_{O}^{\varphi} \geqslant 0, \quad \mathbb{M}_{L}^{\varphi} \geqslant 0, \quad M_{0}^{\varphi}+\sum_{L} M_{L}^{\varphi}>0,
$$

(iii) then the solution $\phi(u)$ (see (6.4)) of the equation (6.2) in a sufficiently small neighbourhood $\bar{\omega}$ of $\overline{\mathrm{K}}$, does exist. It can be obtained by the method of successive aproximations in the norm

$$
-802-
$$


(6.7) $\quad\|u\|_{*}=\sup _{I}\left(\|u\|,\left\|S_{L}^{x} u\right\|\right)$

and is independent of the choice of the $F_{I}$ and $G_{I}$ coefficients. The neighbourhood $\bar{\omega}$ is defined by the inequalities

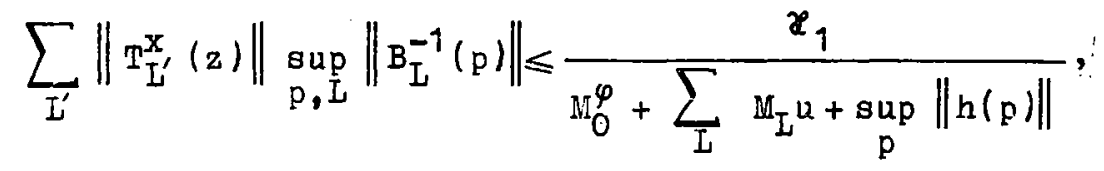

$$
\sum_{I}\left\|T_{I}^{x}(z)\right\| \sup _{x, I}\left\|A_{I}^{-1}(x)\right\| \leqslant \frac{x_{2}}{\pi f_{O}^{f}+\sum_{I} M_{L}^{f}+\|\mu\|},
$$

where $x_{1}+x_{2}=1$.

(iv) $\phi$ is continuous in the norm $\|u\|_{*}$.

P r $0 \circ f$. The following estimation is true:

$$
\begin{aligned}
& \text { (6.10) }\|\phi(u)-\phi(v)\| \leqslant\left\|\sum_{L} T_{L}^{x}(z) F_{L}(p) B_{L}^{-1}(p)\right\| \cdot \\
& \text { - }\left\|\varphi\left(p, u, S_{L}^{X} u\right)-h(p) u-\varphi\left(p, v, S_{L}^{x_{v}}\right)+h(p) v\right\|+ \\
& +\left\|\sum_{I} \mathbb{T}_{I}^{\mathrm{x}}(z) \mathrm{G}_{I}(x) \mathrm{A}_{I}^{-1}(\mathrm{x})\right\| \cdot\left\|\mu u+f\left(x, u, \mathrm{~S}_{\mathrm{L}}^{\mathrm{x}} u\right)-u v-f\left(x, v, S_{L}^{x_{v}}\right)\right\| \leqslant \\
& \leqslant \sum_{L}\left\|T_{L}^{x}(z)\right\| \sup _{p, L}\left\|B_{L}^{-1}(p)\right\|\left(M_{O}^{f}\|L-v\|+\sum_{L} M_{L}^{f}\left\|S_{L}^{x} u-S_{L}^{x} v\right\|\right)+ \\
& +\sup _{p}\|h(p)\| \cdot\|u-v\|+ \\
& +\sum_{I}\left\|\mathrm{~T}_{I}^{\mathrm{x}}(z)\right\| \sup _{x, I}\left\|A_{I}^{-1}(x)\right\|\left(\mathrm{N}_{0}^{f}\|u-v\|+\sum_{I} \mathrm{~N}_{L}^{f} \| \mathrm{S}_{L}^{x_{L}} u-S_{L}^{x} v\right)+
\end{aligned}
$$


18

R.Bitther, Z.Smentek

$$
\begin{aligned}
& +\|\mu\|\|u-\nabla\| \leqslant \sum_{L}\left\|T_{L}^{x}(z)\right\| \sup _{p, L}\left\|B_{i}^{-1}(p)\right\|\left(M_{O}^{\varphi}+\sum_{L} M_{L}+s u p\|h(p)\|\right)+ \\
& +\sum_{I}\left\|T_{I}^{x}(z)\right\| \sup _{x, I}\left\|A_{I}^{-1}(x)\right\|\left(M_{0}^{f}+\sum_{L} M_{L}^{f}+\|\mu\|\left\|_{u}-\nabla\right\|_{*} \cdot\right.
\end{aligned}
$$

Further we have

$$
\begin{aligned}
& \text { (6.11) }\left\|S_{I}^{x} \phi(u)-S_{L}^{x} \phi(v)\right\| \leqslant\left(\sum_{I^{\prime}}\left\|S_{I^{T}}^{T_{I^{\prime}}}\right\| \sup \left\|B_{I}^{-1}(p)\right\|\right) \text {. } \\
& \cdot\left(\mathbf{M}_{0}^{\varphi}+\sum_{\mathrm{I}} \mathrm{M}_{\mathrm{L}}^{\varphi}+\sup _{\mathrm{p}}\|\mathrm{h}(\mathrm{p})\|\right)+ \\
& +\sum_{I}\left\|S_{I}^{X} T_{I}^{X}\right\| \sup _{x, I}\left\|A_{I}^{-1}(x)\right\|\left(M_{0}^{f}+\sum_{L} M_{I}^{f}+\|\mu\|\right)\|u-v\|_{*} \cdot
\end{aligned}
$$

The inequality $(6,10)$ is a particular case of the inequality $(6,11)$ for $L=(0,0, \ldots, 0)$.

The sums $\sum_{I}\left\|\mathrm{~T}_{\mathrm{I}}^{\mathrm{x}}(\mathrm{z})\right\|, \sum_{I}\left\|\mathrm{~T}_{I}^{\mathrm{x}}(\mathrm{z})\right\|, \sum_{I}\left\|\mathrm{~S}_{\mathrm{T}^{\mathrm{x}} \mathrm{I}}^{\mathrm{x}}\right\|$, $\sum_{I}\left\|S_{I^{T}}^{x_{T}}\right\|$, as well as the coefficients $B_{I}^{-1}(p)$ fulfill the conditions $(6.8)$ and $(6.9)$ when $\sup \left\|B_{I}^{-1}(p)\right\|<1$ and the norms $\delta_{1}=\left\|T_{1}\right\|, \ldots, \delta_{m}=\left\|T_{m}\right\|$, are sufficiently small. It results from the inequality $(6.10)$ and $(6.11)$ that $\phi$ is the operation continuous in the norm $\|u\|_{*}$, so we use the method of successive approximations.

$\mathrm{Th}$ e o $\mathrm{r}$ em 5. Let $u$ and $v$ be two solutions of the equation $(6.2)$ in the common space $\overline{\boldsymbol{\Omega}}$ for coefficients $\overline{\mathrm{F}}_{\mathrm{L}}, \overline{\overline{\mathrm{F}}}_{\mathrm{I}}, \overline{\mathrm{G}}_{I}, \overline{\overline{\mathrm{G}}}_{I}$, Then $u=v$. Proof.

$$
\begin{aligned}
u & =\left.\sum_{I} T_{L}^{x}(z) \bar{F}_{L}(p) B_{I}^{-1}(z)\right|_{p=z}\left(\varphi\left(p, u, S_{I}^{x} u\right)-h(p) u\right)+ \\
+ & \left.\sum_{I} T_{I}^{x}(z) \bar{G}_{I}(x) A_{I}^{-1}(z)\right|_{x=2}\left(\mu u+f\left(x, u, S_{I}^{x} u\right)\right) \underset{D f}{=} \bar{\phi}(u) . \\
& =804-
\end{aligned}
$$




$$
\begin{aligned}
\nabla & =\left.\sum_{I} T_{L}^{x}(z) \overline{\bar{F}}_{L}(p) B_{L}^{-1}(z)\right|_{p=z}\left(\varphi\left(p, v, S_{L}^{x} \nabla\right)-h(p) v\right)+ \\
& +\left.\sum_{I} T_{I}^{x}(z) \overline{\bar{G}}_{I}(x) A_{I}^{-1}(z)\right|_{X=2}\left(\mu\left(u+f\left(x, v, S_{L}^{x} v\right)\right)=\overline{D \rho}=\overline{\bar{\phi}}(v) .\right.
\end{aligned}
$$

Then $\|u-v\|_{*}=\|\bar{\phi}(u)-\overline{\bar{\phi}}(v)\|_{*} \leqslant\|\bar{\phi}(u)-\bar{\phi}(v)\|_{*}+$ $+\|\bar{\phi}(v)-\overline{\bar{\phi}}(v)\| \leqslant\left(x_{1}+x_{2}\right)\|u-v\|_{*}+\sup _{p, L}\left\|\bar{F}_{L}(p)-\overline{\bar{F}}_{L}(p)\right\|+$ $+\sup \left\|\bar{G}_{I}(x)-\overline{\bar{G}}_{I}(x)\right\| \cdot\|v\|_{*^{*}}$ Moreover we can take coefficients $\overline{\bar{F}}_{L}(p)$ and $\overline{\bar{G}}_{I}(x)$ for coefficients $\bar{F}_{L}(p)$ and $\bar{G}_{I}(x)$ so that

$$
\begin{aligned}
& \left(\sup _{p, I}\left\|\bar{F}_{L}(p)-\overline{\bar{F}}_{L}(p)\right\|+\sup _{x, I}\left\|\bar{G}_{I}(x)-\overline{\bar{G}}_{I}(x)\right\|\right) \cdot\|v\|_{*} \leqslant \\
& \leqslant\left(1-\left(x_{1}+x_{2}\right)\right)\|u-v\|_{*} \leqslant\|u-v\|_{*} \text {, because } x_{1}+x_{2}<1 .
\end{aligned}
$$

Therefore we must have $u=\nabla$.

\section{About an extension of solutions}

Definition. The function $u^{\prime}=\left\{\left.R\right|_{\Omega^{\prime}}(x)\right\}$ is called a restriction of the function $u=\{R(x)\}_{\Omega}$ from $\Omega$ to $\Omega^{\prime}$.

I $\theta \mathrm{ma}$. If for $\mu_{\alpha}=\{R(x)\}_{\Omega_{\alpha}}, \alpha=1,2, \ldots N$ the equation

$(7.1)$

$$
\sum_{(I, n)} u_{\alpha}=\mu u_{\alpha}+f\left(x, u_{\alpha}, S_{L}^{x} u_{\alpha}\right), \quad x \in \Omega_{\alpha}
$$

with the Iimit condition

(7.2) $\varphi\left(p_{\alpha}, u_{\alpha}, S_{L}^{x} u_{\alpha}\right)=\left.\left.\sum_{I} B_{L}\left(p_{\alpha}\right)\right|_{z=p} x(z) S_{L}^{x} u_{\alpha}\right|_{z=p}+h\left(p_{\alpha}\right) u_{\alpha}$ 
has for each $\alpha=1,2, \ldots, N$ a unique solution $u_{\alpha}$ in $s z_{\alpha}$, and $\Omega_{\alpha} \cap \Omega_{\beta} \neq \phi$ than the equation $(6,2)$, condition $(6,3)$ hes the unique solution $u$ in $\Omega$, where $u:-\left\{u_{\alpha}(x)\right\}$ for $x \in \Omega_{\alpha}, \quad S=\bigcup_{\alpha} \Omega_{\alpha}$.

P $r \circ \circ f^{\alpha}$. The equation (7.1) is considered when $x \in \Omega$, which means $x \in \bigcup_{\alpha} \Omega_{\alpha}$. It has the unigue solution in each of $\Omega_{\alpha}$. In this case these solutions must coincide one with another in $\Omega_{\alpha} \cap \Omega_{\beta} \neq \phi$, which leads to the common solution $u$ in $\Omega=\bigcup \alpha \Omega_{\alpha}$. This solution is the only one in $\Omega$, because if $u$ and $\bar{u}$ were different in $\Omega$, then we would have $\mathrm{u}=\overline{\mathrm{u}}$ in $\Omega_{\alpha}$ for $\alpha=1,2, \ldots, \mathrm{N}$, which means $\mathrm{u}=\overline{\mathrm{u}}$. C O I O II a r y. If $\left\|\left(T^{x}\right)_{1}(q)\right\|_{\alpha}<\delta$, where $\delta$ fuIfils the condition $(6.6)$ and $\Omega_{\alpha}$ is the set from the lemma, in this case there is exactly one solution in the sum $\bigcup_{\alpha} \Omega_{\alpha}$ in the (7.1) equation. This solution can be obtained using the method of succesive approximation.

$\mathrm{E} x \mathrm{ample}$ 1. The equation

$$
x \frac{\partial^{3} u}{\partial x^{2} \partial y}+x^{2} y^{2} \frac{\partial^{2} u}{\partial x^{2}}=f(x, y), \quad(x, y) \in D,
$$

where $D=\left\{(x, y): x^{2}+y^{2}<1, \quad x, y>\alpha>0\right\}, \quad s_{1}^{x}=\frac{\partial}{\partial x}$, $s_{2}^{y}=\frac{\partial}{\partial y}$ leads as in the formula $(6.2)$ to the equation

$$
\text { (7.4) }\left.\left.\right|_{\substack{z=x \\ w=y}} z\right|_{\substack{x=z \\ y=w}}\left(S_{1}^{x}\right)^{2} S_{2}^{y} u+\left.\left.\right|_{\substack{z=x \\ w=y}} z^{2} w^{2}\right|_{\substack{y=z \\ y=w}} ^{\prime}\left(S_{1}^{x}\right)^{2} u=f(x, y) .
$$

The limit condition

$$
\left.x_{0} \frac{\partial u}{\partial x}\right|_{\left(x_{0}, y_{0}\right)}=u\left(x_{0}, y_{0}\right)
$$

for $x_{0}^{2}+y_{0}^{2}=1, x_{0}, y_{0}>\alpha>0$ give the equation

$(7.6)$

$$
\left|\begin{array}{l|l}
p=x_{0} \\
r=y_{0}
\end{array}\right| \begin{aligned}
& x_{0}=p \\
& y_{0}=r
\end{aligned}
$$


on the boundary $\Gamma=\left\{\left(x_{0}, y_{0}\right): x_{0}^{2}+z_{0}^{2}=1, x_{0}, y_{0}>\alpha>0\right\}$ of domain D.

By Theorem 3, the solution $u$ can be written in the formula $(6.4)$, if

$$
\begin{aligned}
T_{1}^{x}(z) & =\int_{z}^{x}, T_{y}^{2}(w)=\int_{w}^{J},\left\{(x, J, z, w): x^{2}+J^{2}<1, x, J>\alpha>0\right\} \\
(7.7) u & \left.=\left.T_{1}^{x}\left(x_{0}\right)\right|_{\substack{z=x_{0} \\
w=y_{0}}} F_{1,0}(z, w) \frac{1}{z} u(z, w)+T_{1}^{x}(z)\right)^{2} T_{2}^{y}(w) \frac{1}{z} G_{2,1}(z, w) u(z, w)+ \\
& +\left(T_{1}^{x}(z)\right)^{2} \frac{1}{z^{2} w^{2}} G_{2,0}(z, w) u(z, w) \underset{D f}{=} \phi(u),
\end{aligned}
$$

where

$F_{1,0}\left(x_{0}, y_{0}\right)=1, G_{2,1}(x, y)+G_{2,0}(x, y)=1, G_{2,1}, G_{2,0} \geqslant 0$.

Further we use Theorems 2 and 3.

For solution (7.7) we have

$$
\begin{aligned}
\|\phi(u)-\phi(v)\| & \leqslant\left(\sup \left\|x-x_{0}\right\| \frac{1}{\|z\|}+\sup \frac{\|x-2\|^{2}}{2}\|s-w\|+\right. \\
& +\sup \|x-z\| \frac{1}{\left\|z^{2} w^{2}\right\|}\|u-v\| .
\end{aligned}
$$

Let the set $D_{0}$ be defined by the following formula

$$
\begin{aligned}
D_{0}= & \left\{(x, y): \sup _{z=x_{0}}\left\|x-x_{0}\right\| \frac{1}{\|z\|}<\frac{x}{3}, \text { sup } \frac{\|x-z\|^{2}}{2}\|y-w\|<\frac{x}{3},\right. \\
& \left.\sup \|x-z\| \frac{1}{\left\|z^{2}{ }^{2}\right\|}<\frac{x}{3}\right\} .
\end{aligned}
$$

Then in the set $D_{0},\|\phi(u)-\phi(v)\| \leqslant x\|u-v\|$ añu if $0<x<1$ we can use the method of succesive approximations:

We obtain some neighbourhood $D_{0} \subset D$ of the boundary $\Gamma$ in which the equation (7.3) with the condition (7.5) has a so- 
lution. Taking another equations we can have even $D=D_{0}$, if $\alpha$ is so large that the eatimation $\|\varphi(u)-\varphi(v)\| \leq x\|u-v\|$ for $0<\boldsymbol{r}<1$ is fulfilied at all points of $D$.

\section{B I B I I O G R A P H Y}

$[1]$ I. B e I $\mathrm{g}$ : Solution of simultaneous equations. Demonstratio Wath. 10 (1977) 515-522.

$[2]$ R. B it t n e $r$ : Operational calculus in linear spaces. Studia Wath. 20 (1961) 1-18.

$[3] R_{\text {. B }} i t \mathrm{t}$ e $r$, Z. S mentek: Taylor's formula for many derivatives. First integrals. Preprint No.5, April 1978, University of Gidańsk, Institute of Mathematics.

$[4]$ D. Prze worska-Rolewicz: Algebraic theory of right invertible operators. Studia Wath. 48

[ (1973) 129-144.

[5]D. Prze worska-Rolewicz: Admissible initial operators for superpositions of right invertible operators. Ann. Polon. Math. 33 (1976) 113-120.

INSTITUTE OF MATHEMATICS, UNIVERSITY, GDANSK Received June 8, 1977. 